\title{
Perception de risques du paludisme et utilisation des moustiquaires au Tchad
}

\author{
Fissou Henry YANDA I ${ }^{1 *}$, Kebfene MOUNDINE ${ }^{2}$, Ephraïm DJOUMBE ${ }^{2}$, \\ Kodbesse BOULOTIGAM ${ }^{2}$, Azoukalne MOUKENET ${ }^{2}$, Israel DEMBA KODINDO ${ }^{2}$ et \\ Clement KERAH HINZOUMBE ${ }^{2}$
}

\author{
${ }^{I}$ Laboratoire de l'Hôpital de la Mère et de l'Enfant de N'Djamena (HME), Tchad. \\ ${ }^{2}$ Programme National de Lutte contre le Paludisme (PNLP), Tchad. \\ *Auteur correspondant, E-mail : fissouhenry@yahoo.fr; Tel : 0023563772580.
}

\section{RESUME}

L'utilisation des Moustiquaires Imprégnées d'Insecticide à Longue Durée d'Action (MILDA) et la chimio - prévention du paludisme sont des stratégies efficaces de lutte contre le paludisme. L'objectif de cette étude est d'évaluer le niveau de connaissance de la communauté sur le paludisme et l'utilisation des MILDA. Une étude descriptive a été effectuée au Tchad, sur la base des questionnaires administrés aux chefs de ménages. Les données collectées ont été saisies à l'aide du logiciel Acces 2007, nettoyées et validées au cours d'un atelier à N'Djamena. Des 2000 personnes enquêtés, 1388 (69,4\%) réponses ont été satisfaisantes selon les critères utilisés. Un taux de $80,6 \%$ des enquêtés déclarent disposer des MILDA, et 37,6\% de ce taux affirment les avoir utilisées la nuit précédant l'enquête. Cependant, $68,2 \%$ des répondants perçoivent que le paludisme est dangereux pour le foetus et les femmes enceintes. Aussi, $50.4 \%$ d'individus affirment dormir sous la MILDA à partir de 20 heures. Cette étude a montré que malgré que le paludisme soit la première cause de consultations médicales au Tchad, certaines personnes ne perçoivent pas les risques de cette maladie. Plus de la moitié des personnes disposant des MILDA ne les ont pas utilisés à la veille de l'enquête.

(C) 2016 International Formulae Group. All rights reserved.

Mots clés : Paludisme, connaissance, moustiquaire, utilisation, Tchad.

\section{Perception of malaria risk and use of bed nets in Chad}

\begin{abstract}
The use of Long Lasting Insecticidal-treated Nets (LLINs) and the seasonal malaria chemoprevention are efficient strategies in the fight against malaria. The objective of this survey is to assess the community knowledge about malaria and LLIN use. A descriptive households' survey has been conducted in Chad using questionnaires targeting the head of households. Data collected were entered in Access 2007, cleaned and validated during a workshop held in N'Djamena. Of the 2,000 people targeted, $1,388(69.4 \%)$ responses satisfied all the criteria. $80.6 \%$ of the respondents declared owning a LLIN but only $37.6 \%$ of those state having used it the night prior to the interview. $68.2 \%$ of the respondents perceive malaria as dangerous for the
\end{abstract}


fetus and the pregnant women. $50.4 \%$ of the respondents state sleeping under the LLIN starting at 8 pm. This survey shows that despite being the first cause of out-patient visits in Chad, risks related to malaria are not fully perceived by the population. More than half of the respondents owning a LLIN did not sleep under it the night prior to the survey.

(C) 2016 International Formulae Group. All rights reserved.

Keywords: malaria, knowledge, mosquito net use, Chad.

\section{INTRODUCTION}

Le paludisme constitue un véritable problème de santé publique dans le monde et particulièrement en Afrique subsaharienne (Buonsenso et Cataldi, 2010 ; Unicef, 2013 ; Lundblom et al., 2013; Kilama, 2005). Au Tchad, de 2000 à 2012, la mortalité due au paludisme a été estimée à $42 \%$ atteignant $48 \%$ chez les enfants de moins de 5 ans. Les cas suspects de paludisme sont passés de 528.454 en 2011 à 1.272.841 en 2013 (PNLP, 2013) puis à 1.513 .772 cas en 2014 avec 1.720 décès (PNLP, 2014). L'analyse des données de la surveillance épidémiologique a montré que la transmission du paludisme est saisonnière dans la bande sahélienne. Pour réduire le fardeau de cette maladie, la ChimioPrévention du Paludisme Saisonnier (CPS) a été recommandée par l'Organisation Mondiale de la Santé (OMS) en 2012 dans toute la sousrégion du Sahel où la transmission est courte et ne dépassant pas quatre mois (OMS, 2015). La CPS consiste à administrer de façon intermittente, les traitements complets par un médicament antipaludique ( $\mathrm{AQ}+\mathrm{SP})$ aux enfants de 3 à 59 mois au cours de la saison de haute transmission du paludisme pour éviter la maladie et ceci, en maintenant des concentrations thérapeutiques en médicament antipaludique dans le sang pendant toute cette période (OMS, 2015). Au Niger, 54,82\% d'enfant et $48,21 \%$ des femmes enceintes ont dormi sous moustiquaire la nuit précédant une enquête (Thwing et al., 2008). Au Sénégal, environ trois enfants sur dix dorment sous MILDA (Ndiaye et al., 2007). Le Tchad a adopté cette stratégie dans sa politique nationale et dans son plan stratégique 2014-
2018 (PNLP, 2014). Par ailleurs, il est établi que l'usage convenable de MILDA permet de prévenir la transmission de la maladie. Des campagnes de distribution gratuite de MILDA ont été effectuées sur l'ensemble du territoire et en particulier dans les zones de forte endémicité. Chaque ménage a bénéficié d'une MILDA pour deux personnes. Cependant, certains auteurs pensent que le manque de connaissance cognitive d'un objet technique peut influencer sur les pratiques d'usabilité (Le Coadic et al., 2004). C'est dans cette perspective que le présent travail s'inscrit. Le but visé par cette étude est d'évaluer la perception des risques du paludisme au niveau communautaire, et les attitudes pratiques visà-vis de l'utilisation des moustiquaires dans les ménages.

\section{MATERIEL ET METHODES}

\section{Type d'étude et zone d'enquête}

Il s'agit d'une étude descriptive transversale sur une période de 2 mois allant du 15 avril au 15 juin 2015. L'enquête s'est déroulée dans huit (8) Districts Sanitaires à savoir: Dourbali, Mandélia, Massakory, Massaguet, Ati, Oum-Hadjer, Mao et Rig-Rig. 4 participants à la CPS 2015 et 4 autres nonparticipants. Toutes ces zones ont bénéficié des campagnes de distribution gratuite des MILDA (Figure 1).

\section{Technique d'échantillonnage}

La méthode d'échantillonnage par grappe à trois degrés a été requise. Au premier degré, grâce au logiciel de statistiques $\mathrm{R}$. Quatre (4) districts ont été tiré au hasard parmi les bénéficiaires (DSP) de la CPS en 
2015 et quatre (4) autres issues de la zone éligible pour la CPS mais non participants à la CPS en 2015 (DSNP). Au deuxième degré, sur la base de sondage des Zones de Responsabilité (ZR) des districts choisis, il a été échantillonné aléatoirement $10 \%$ des ZR qui ont constitué des Zones de Dénombrement (ZD). La clé de répartition des échantillons par district était le poids démographique. Ensuite, la taille de l'échantillon calculée pour le district a été rapportée au nombre de ménages dénombrés. Ce qui a permis ainsi, d'avoir un pas de sondage. La méthode de " Stylo » indiquait la direction aux enquêteurs une fois dans la zone de dénombrement. Cette méthode de «stylo » n'était applicable qu'à la zone A (c'est-à-dire en zone urbaine). Dans la zone $\mathrm{B}$ (au-delà de $5 \mathrm{~km}$ ) considérée comme zone rurale, le pas de sondage était inapplicable $\mathrm{du}$ fait de la configuration spatiale particulière des villages. Les pasteurs nomades ayant installé leurs camps de passage provisoires autour des villages constituant ainsi des regroupements sporadiques des ménages, la méthode aréolaire a été requise. Ainsi, l'aréole choisie était enquêtée systématiquement.

\section{Collecte des données}

Les données ont été collectées sur la base des questionnaires, par interview directe des personnes à leur domicile. Ce questionnaire contenait des informations relatives aux caractéristiques sociodémographiques (âge, sexe, niveau d'instruction, situation matrimonial, mode de vie), la perception et la connaissance du risque de paludisme, la possession et l'utilisation des moustiquaires par les ménages.

\section{Traitement des données}

Une base de donné a été conçue sous Acces 2007. La saisie informatique des données a été faite au cours d'un atelier par une équipe de saisie et de traitement. Une semaine durant, les données ont été saisies, nettoyées, contrôlées et validées par l'équipe dédiée. La saisie des données et sa validation ont respecté les procédures de contrôle qualité. Les modalités d'accès sont réglementées conformément aux lois sur les libertés et informatiques. Le stockage a été fait sur des serveurs externalisés (Hard Disque).

\section{RESULTATS}

$\mathrm{Au}$ total 2000 questionnaires ont été administrés aux parents. De ce nombre, 1388 questionnaires ont été jugés satisfaisantes selon le critère utilisé. Par contre, 612 fiches sont incomplètes. Le taux de réponse exhaustif est de $69,4 \%$.

\section{Caractéristiques sociodémographiques des ménages enquêtés}

Le Tableau 1 montre les caractéristiques sociodémographiques des personnes interviewées. Au total $85,3 \%$ des ménages enquêtés sont dirigés par des hommes et $14,7 \%$ par des femmes. Les chefs de ménage sont en majorité monogames $(82,1 \%)$. Un taux de $52,3 \%$ des parents enquêtés sont actifs à temps partiels contre $22,4 \%$, occupés à temps plein dans l'élevage pastoral. Les enquêtés ont pour la plupart, un mode de vie sédentaire $(99,1 \%)$. Parmi ces personnes, $62,40 \%$ des pères et $71,3 \%$ des mères non aucun niveau de scolarité.

\section{Perception de risque du paludisme}

Un taux de $60,24 \%$ des répondants affirme que le paludisme est une maladie dangereuse et que leurs enfants pourraient le contracter. Mais 54,63\% seulement sont conscients de leurs risques personnels. En revanche, 56,33\% des personnes interrogées sont d'accord que leurs enfants pourraient mourir du paludisme alors qu'elles sont $70,72 \%$ à être conscientes de leurs risques personnels de décès par suite de paludisme. $68,2 \%$ des répondants sont d'accord que le paludisme est dangereux pour le fotus et 
même pour la femme enceinte $(68,18 \%)$. Ils sont $82,35 \%$ à percevoir le danger que représente le paludisme pour les femmes non enceinte et $71,4 \%$ pour toutes les femmes.

\section{Possession et utilisation des moustiquaires}

Sur l'effectif global, $80,6 \%$ des ménages enquêtés déclarent disposer d'une MILDA pour 2 personnes. Mais, seulement $37,6 \%$ des ménages reconnaissent les avoir utilisés la nuit précédant l'enquête. En ce qui concerne l'utilisation des moustiquaires chez les enfants de moins de 5 ans, $41,8 \%$ n'ont pas dormi sous une moustiquaire la nuit précédant l'enquête. S'agissant de la qualité de l'utilisation des MILDA, 32,6\% déclarent allez sous une MILDA après 20 heures, 17,8\% après 22 heures, et $49,3 \%$ au crépuscule à partir de 18 heures.

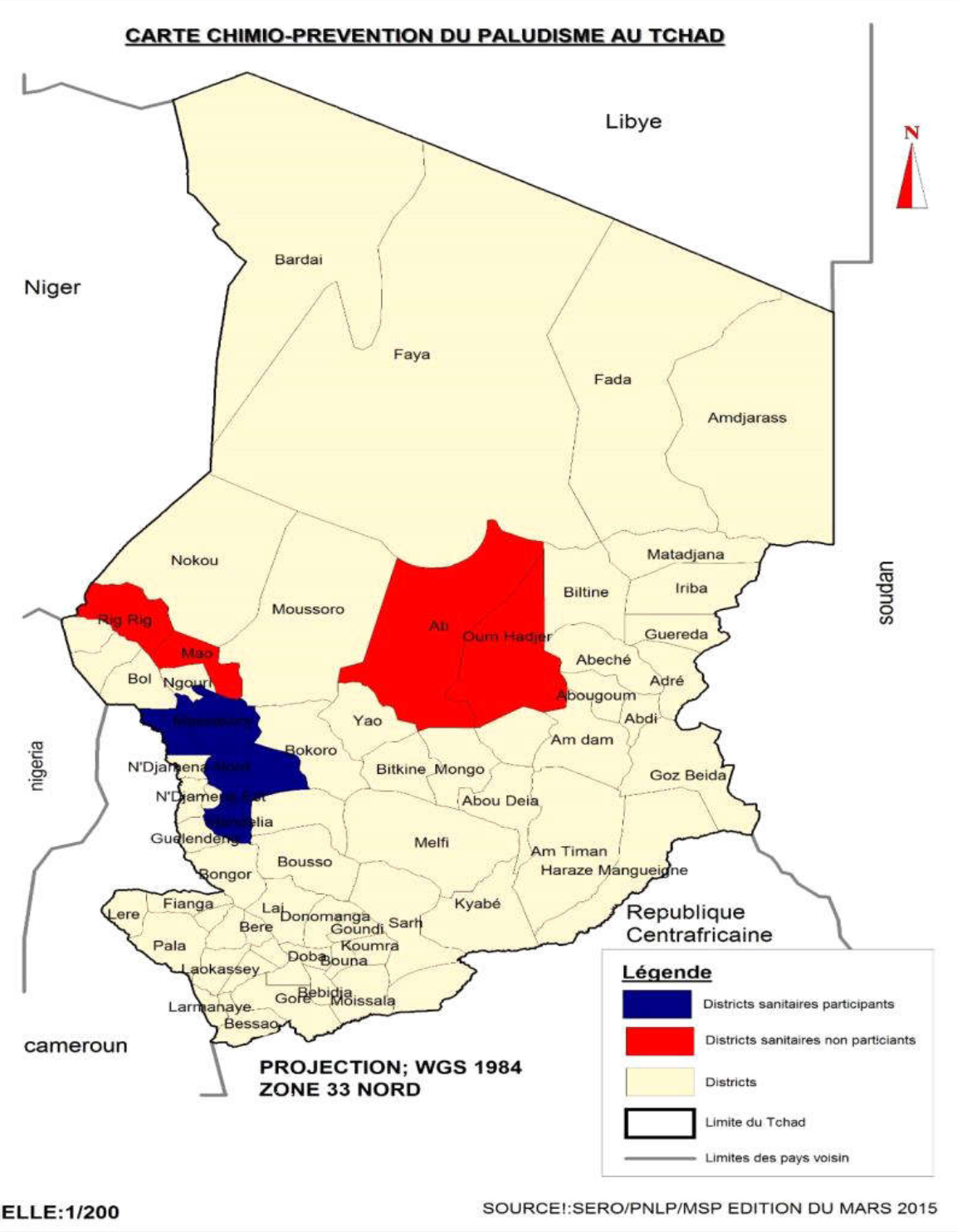

Figure 1 : Carte de chimio-prévention du paludisme au Tchad. 
Tableau 1 : Caractéristiques sociodémographiques des personnes enquêtées.

\begin{tabular}{lll}
\hline Variables & Effectif (n) & Pourcentage \\
\hline Sexe & & \\
Femme & 204 & $14,7 \%$ \\
Homme & 1184 & $85,3 \%$ \\
Statut matrimonial du chef de ménage & & \\
Monogame & 1139 & $82,1 \%$ \\
Polygame & 249 & $17,9 \%$ \\
Statut socioprofessionnel & & \\
Actif à temps plein & 309 & $22,3 \%$ \\
Actif à temps partiel & 726 & $52,3 \%$ \\
Chômeur & 186 & $13,4 \%$ \\
Inactif & 167 & $12,0 \%$ \\
Mode de vie des parents & & \\
Nomade & 12 & $0,9 \%$ \\
Sédentaire & 1378 & $99,1 \%$ \\
Total & 1841 & $100,00 \%$ \\
\hline
\end{tabular}




\section{DISCUSSION}

\section{Connaissance de risques du paludisme}

Cette étude révèle que plus de $60 \%$ des personnes enquêtées sont conscientes qu'elles-mêmes ou leurs enfants pourraient mourir de paludisme. De nombreux répondants $(68,2 \%)$ sont d'accord que le paludisme est dangereux pour le foetus et même pour la femme enceinte $(68,18 \%)$. Cependant, $30 \%$ d'individus ignorent parfaitement les risques liés au paludisme. Au Nigéria une enquête a montré que $74 \%$ des personnes interrogés avaient une connaissance sur le paludisme. C'est aussi le cas des enquêtes du Soudan (Elfatih et al., 2006) et du Nigéria (Okafor et Obu, 2004). Par contre au Sénégal une étude a rapporté un taux de connaissance du paludisme plus élevé que celui du Tchad. Cette étude a montré que $80 \%$ des Sénégalaises connaissaient les risques liés au paludisme. Cette différence de taux entre ces deux pays pourrait s'expliquer par la différence d'Indice de Développement Humain (IDH). Il faut rappeler que le Tchad est l'un des pays ayant un Indice de Développement Humain plus bas $(0,340)$ comparé à celui du Sénégal $(0,470)$ (IDH, 2012). Ces observations corroborent également les points de vue de nombreux auteurs qui soutiennent qu'il y a une influence de l'instruction sur les pratiques au niveau mental et social. Le niveau d'éducation de la mère a une influence sur l'utilisation de la MILDA (Cilundika et al., 2016). L'instruction a un effet positif sur l'adoption de comportements novateurs, la compréhension des messages sanitaires. Au Sénégal, l'instruction a été citée comme un des facteurs déterminant de l'utilisation des moustiquaires. Les personnes qui savaient lire et écrire avaient une meilleure connaissance sur le paludisme que les non scolarisées (Seck et al., 2008 ; Tall-Dia et al., 2002).

\section{Possession des MILDA}

$\mathrm{La}$ population tchadienne est très favorable à la possession des MILDA. Quasiment tous les ménages enquêtés disposent des MILDA. Un résultat similaire a été observé également au Mali (Jenny et al., 2008). Cet auteur rapporte que $80,6 \%$ des Maliens possèdent des MILDA. Toutefois, les taux obtenus au Tchad et au Mali sont supérieurs à ceux rapportés par Faye (2008) $(76,7 \%)$ et l'enquête CDC (65\%) au Niger (Thwing et al., 2008). Il faut noter qu'au Tchad, la distribution des MILDA est fortement décentralisée et gratuite. La dernière distribution s'est faite en 2014 par l'organisation d'une campagne d'un mois dans tout le pays. Pendant cette campagne, une MILDA a été donnée pour deux personnes par ménage. Actuellement dans les différents centres de santé, chaque femme enceinte bénéficie d'une MILDA au premier contact de la consultation préventive. Tout nouveau-né vu à la première vaccination de routine bénéficie d'une MILDA. L'ensemble de ces stratégies ont permis à de nombreux ménage de posséder des MILDA.

\section{Utilisation des MILDA}

Il se pose un problème d'utilisation de MILDA observé dans cette étude. Un écart important a été constaté entre le taux de possession d'une MILDA et son utilisation. En effet, la moitié des individus possédant des MILDA (37,6\%) affirment ne pas dormi sous la MILDA la nuit précédant l'enquête. Il en est de même pour les enfants. Ces observations corroborent les études des autres pays d'Afrique (Iloh et al., 2013 ; Cilundika et al., 2016 ; Olusegun-Joseph et al., 2016). Dans les pratiques courantes, les moustiquaires ne sont pas installées en permanence dans la plupart de ménage. Les MILDA ne semblent pas être perçu par la communauté comme outil de lutte efficace contre le paludisme, mais plutôt moyen de lutte contre la nuisance occasionnée 
par les moustiques (Doannio et al., 2004). Une étude rapporte que l'inadaptation des moustiquaires à l'environnement tel que l'espace très restreint, ou le lit en bambou pourrait expliquer l'écart entre le taux de possession des moustiquaire et l'utilisation (Doannio et al., 2006). Une autre étude a permis de constater que certains ménages n'utilisant pas les MILDA mais préféraient acheter des aérosols, des serpentins fumigènes (Wiseman et al., 2006). Ces observations ne sont quasiment pas valables pour le Tchad du fait de la distribution gratuite des MILDA dans toutes les régions.

$\mathrm{Au}$ Tchad, la non-utilisation des MILDA serait liée au déficit de connaissance sur le sujet. La sensibilisation de la communauté sur l'étiologie du paludisme et l'utilité des MILDA comme meilleur outil de prévention du paludisme pourrait augmenter la visibilité de la population sur le sujet et de ce fait les motiver à la bonne utilisation. Selon Ndour et al. (2006), la communication pour le changement de comportement bien planifié et adapté au contexte locale pourrait avoir un impact positif sur l'utilisation de cet outil.

En ce qui concerne la période d'utilisation des MILDA, cette étude a permis de constater que $50,4 \%$ de personnes vont sous MILDA entre 20 et 22 heures. Par contre $49,3 \%$ d'individus déclarent allez sous MILDA au crépuscule à partir de 18 heures. Ces données laissent présager quelques attitudes favorables à la prévention du paludisme car les moustiques son actif à partir de 20 heures et la plupart d'anophèle pique autour de 22 heures.

\section{Conclusion}

Bien que le paludisme soit la première cause de consultation médicale au Tchad, les populations exposées ne sont pas toutes conscientes du risque de la maladie. Une moitié des personnes disposant des MILDA ne l'ont pas utilisé pendant la nuit qui a précédé l'enquête. Les informations sur l'utilisation convenable de cet outil ne sont pas bien connues par la communauté. Il apparait capital d'utiliser des dialectes adaptés à chaque localité dans les différents programmes de sensibilisation à base communautaire en vue d'améliorer leur connaissance en matière de paludisme. Une enquête qualitative sur les raisons de nonutilisation des MILDA serait utile pour permettre d'expliquer les représentations socioculturelles et les perceptions limitant leur taux d'utilisation.

\section{CONFLIT D'INTERETS}

Les auteurs déclarent ne pas avoir de conflit d'intérêts.

\section{CONTRIBUTIONS DES AUTEURS}

FHY a participé à l'élaboration du sujet et l'écriture du manuscrit; KM a conduit la recherche, la saisie et l'analyse des données. $\mathrm{KB}, \mathrm{ED}, \mathrm{AM}, \mathrm{IK}$ et $\mathrm{CKH}$ ont participé à l'enquête et à la correction du manuscrit.

\section{REMERCIEMENTS}

Nous remercions le Ministère de la Santé Publique du Tchad et ses partenaires pour l'appui financier, et les personnes interviewées qui ont sacrifié de leur temps pour participer à l'enquête.

\section{REFERENCES}

Buonsenso D, Cataldi L. 2010. Watch out for malaria : still a leading cause of child death worldwide. Italian Journal of Pediatrics, 36: 58.

Cilundika MP, Nyota NO, Oscar LN. 2016. Problématique de l'utilisation des Moustiquaires Imprégnées d'insecticide à Longue Durée (MILD) chez les enfants de moins de 5 ans en République Démocratique du Congo. Pan. Afr. Med. J., 23: 101.

Doannio JMC, Konnan YL, Amalaman K, Attiah J. 2004. Connaissances, attitudes et pratiques des populations vis-à-vis des 
moustiques dans la zone urbaine et périurbaine de Bouake et dans les villages de Kafine et Kabolo. Anthropologie Médicale, Bull. Soc. Pathol. Exot., 97(4): 295-301.

Doannio JMC, Doudou DT, Konan LY, Djouaka R, Pare TL, Baldet T, Akogbeto M, Monjour L. 2006. Représentations sociales et pratiques liées à l'utilisation des moustiquaires dans la lutte contre le paludisme en Cote d'Ivoire (Afrique de l'Ouest). Med. Trop., 66: 45-52.

Elfatih MM, Kamal H, Salah HA, Eldirdieri SA, Khalid AM. 2006. Treatment-seeking behaviour for malaria in children under five years of age : implication for home management in rural areas with high seasonal transmission in Sudan. Malar. J., 5: 60-5.

Faye SL. 2012. Comprendre la non-utilisation des moustiquaires imprégnées à longue durée d'action (MILDA) au Niger Understanding the non-use of long-lasting impregnated nets (LLINs) in Niger. Med. Sante Trop., 22: 203-209.

Iloh GU, Amadi AN, Obikwu CE. 2013. Family biosocial variables driving adherence to the use of insecticide treated nets among under-five children managed for malaria in a rural hospital in eastern Nigeria. Niger J. Med., 22(1): 37-44.

Indice de Développement Humain (IDH). 2012. Wikimédia. Foundation, Inc.

Jenny C, Peter B, Rachelle D, Jo-Anne M, Manisha K. 2008. Rapport d'Évaluation de la possession et de l'utilisation des moustiquaires imprégnées de l'insecticide long durée (MILDs) au Mali huit mois après la campagne intégrée de décembre 2007. Health Bridge, 47p.

Kilama WL. 2005. Ethical prospective on malaria research for Africa. Acta. Trop., 95: 276 - 284.

Le Coadic Y. 2004. Usages et usagers de l'information, Armand Colin (ed). Paris, p. 127.
Lundblom K1, Murungi L, Nyaga V, Olsson D, Rono J, Osier F, Ogada E, Montgomery S, Scott JA, Marsh K, Färnert A. 2013. Plasmodium falciparum infection patterns since birth and risk of severe malaria: a nested case-control study in children on the coast of Kenya. PLoS One. Pub.Med., 8(2): e56032.

Ndiaye S, Ayad A. 2007. Enquête nationale sur le paludisme au Sénégal. Macro International (ed), Caliverton, p. 71.

Ndour CT, Ba O, Manga NM, Fortes ML, Nyamwasa D, Sow PS. 2006. Le paludisme : connaissance, attitudes et pratiques des chefs de ménage de la population rurale de Gossas, Sénégal. Bull. Soc. Pathol. Exot., 99(4) : 290-293.

Okafor HU, Obu HA. 2005. Caregivers's knowledge, attitude and practice on childhood malaria and treatment in urban and rural communities in Enugu, southeast Nigeria. Public Health, 119(5): 409 414.

OMS. 2015. WHO policy recommendation: Seasonal malaria chemoprevention (SMC) for Plasmodium falciparum malaria control in highly seasonal transmission areas of the Sahel subregion in Africa [Internet]. WHO. [cited 2015 May 28].

Olusegun-Joseph TS, Oboh MA, Uduak MU. 2016. A survey of malaria prevalence and antimalarial preventive measures amongst students of university of lagos, nigeria. Afr. J. Cln. Exper. Microbiol., 17(4): 267273.

PNLP (Programme National de Lutte contre le Paludisme). 2013. Rapport annuel d'Activités. Ministère de la Santé Publique, 27p.

PNLP (Programme National de Lutte contre le Paludisme). 2014. Plan Stratégique 20142018. Ministère de la Santé Publique, $39 \mathrm{p}$.

Seck I, Fall IS, Faye A, Ba O, Tal-Dia A. 2008. Connaissances, attitudes et 
pratiques des femmes sur le paludisme, dans la zone rurale de Poponguine, Sénégal. Med. Trop., 68: 629-633.

Tall-Dia A, Fall IS, Camara B, Wone I, Ndiaye P, Mbaye, Diouf FN. 2002. Obstacle dans la prise en charge du paludisme de l'enfant dans la ville de Mékhé (Sénégal). Dakar Med., 47: 15963.

Thwing J, Hochberg N, Vanden Eng J, Issifi S, Eliades MJ, Minkoulou E, Wolkon A, Gado H, Ibrahim O, Newman
RD, Lama M. 2008. Insecticide-treated net ownership and usage in Niger after a nationwide integrated campaign. Trop. Med. Int. Health., 13(6): 1-8.

Unicef. 2013. La situation des enfants dans le monde en 2012. Unicef.

Wiseman V, McElroy B, Conteh L, Stevens W. 2006. Malaria prevention in The Gambia: patterns of expenditure and determinants of demand at the household level. Trop. Med. Int. Health, 11: 419431. 